\title{
Qweak: A Precision Measurement of the Proton's Weak Charge
}

\author{
Gregory S. Mitchell, for the Qweak Collaboration ${ }^{\dagger}$ \\ Physics Division, Los Alamos National Laboratory, Los Alamos, NM 87545, USA
}

\begin{abstract}
The Qweak experiment at Jefferson Lab aims to make a $4 \%$ measurement of the parity-violating asymmetry in elastic scattering at very low $Q^{2}$ of a longitudinally polarized electron beam on a proton target. The experiment will measure the weak charge of the proton, and thus the weak mixing angle at low energy scale, providing a precision test of the Standard Model. Since the value of the weak mixing angle is approximately $1 / 4$, the weak charge of the proton $Q_{w}^{p}=1-4 \sin ^{2} \theta_{w}$ is suppressed in the Standard Model, making it especially sensitive to the value of the mixing angle and also to possible new physics. The experiment is approved to run at JLab, and the construction plan calls for the hardware to be ready to install in Hall $\mathrm{C}$ in 2007 . The experiment will be a 2200 hour measurement, employing: an $80 \%$ polarized, $180 \mu \mathrm{A}, 1.2$ $\mathrm{GeV}$ electron beam; a $35 \mathrm{~cm}$ liquid hydrogen target; and a toroidal magnet to focus electrons scattered at $9^{\circ}$, a small forward angle corresponding to $Q^{2}$ $=0.03(\mathrm{GeV} / \mathrm{c})^{2}$. With these kinematics the systematic uncertainties from hadronic processes are strongly suppressed. To obtain the necessary statistics the experiment must run at an event rate of over $6 \mathrm{GHz}$. This requires current mode detection of the scattered electrons, which will be achieved with synthetic quartz Cerenkov detectors. A tracking system will be used in a low-rate counting mode to determine average $Q^{2}$ and the dilution factor of background events. The theoretical context of the experiment and the status of its design are discussed.
\end{abstract}

\section{INTRODUCTION}

There are strong theoretical reasons to expect that the Standard Model is a lowenergy effective theory of some more fundamental description of nature. To identify new physics, one method is to observe directly new particles and interactions at large energy scale. Alternatively, an indirect search can be made at low energy, where a precision measurement may observe small effects due to the new physics.

The Qweak experiment [1] at Jefferson Lab (JLab) will make such a precision measurement of the asymmetry between cross-sections for positive and negative helicity electrons in polarized elastic electron-proton scattering. The asymmetry violates parity, and arises from the interference of electromagnetic and weak amplitudes (photon and $Z$ boson exchange). At this low energy scale, the asymmetry is a measure of the weak charge of the proton, $\mathrm{Q}_{w}^{p}$, which is the strength of the weak vector coupling of the $Z$ boson to the proton. In the limit of small scattering angle and small momentum transfer $\left(Q^{2} \rightarrow 0\right)$, the asymmetry is given by [2]:

$$
\frac{\sigma_{+}-\sigma_{-}}{\sigma_{+}+\sigma_{-}}=\left[\frac{-G_{F}}{4 \pi \alpha \sqrt{2}}\right]\left[Q^{2} \mathrm{Q}_{w}^{p}+Q^{4} B\left(Q^{2}\right)\right] \approx-0.3 \text { ppm at } Q^{2}=0.03 \mathrm{GeV}^{2}
$$




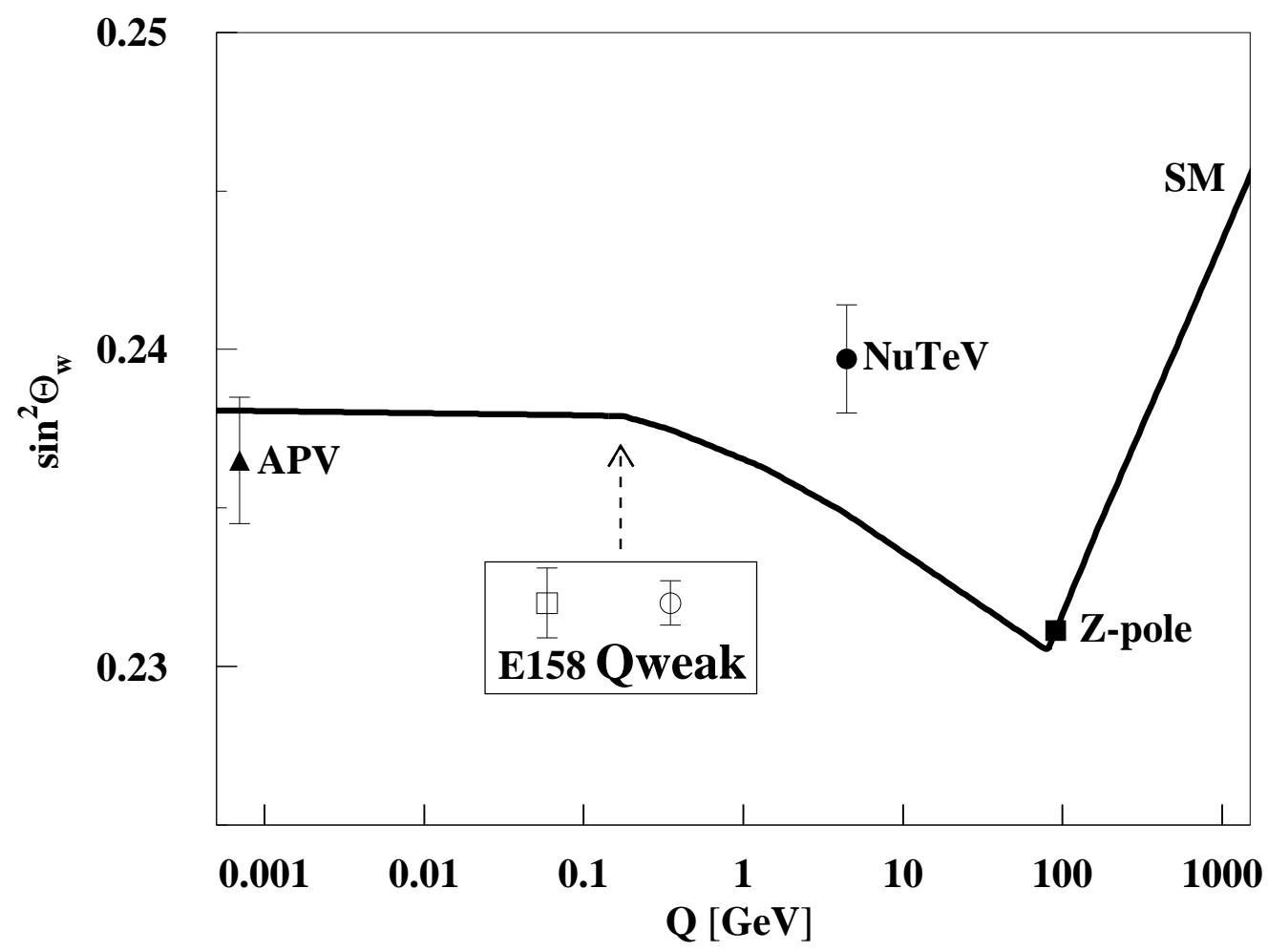

Figure 1: Running of the weak mixing angle in the Standard Model, calculated in the $\overline{\mathrm{MS}}$ scheme. Shown are results from atomic parity violation $[6,7,8], \mathrm{NuTeV}[11]$, and the $Z$ pole [5]. E158 [12] is a currently running experiment at SLAC, and the error bar shown is the E158 proposal goal. Figure courtesy of J. Erler, A. Kurylov, and M.J. Ramsey-Musolf [3].

where $B\left(Q^{2}\right)$ is a contribution from electromagnetic and weak form factors. To lowest order, the weak charge is $\mathrm{Q}_{w}^{p}=1-4 \sin ^{2} \theta_{w}$, where $\sin ^{2} \theta_{w} \approx 0.23$ is the weak mixing angle. The goal of the Qweak experiment is a $4 \%$ measurement of $\mathrm{Q}_{w}^{p}$, which corresponds to a $0.3 \%$ measurement of $\sin ^{2} \theta_{w}$.

The weak mixing angle is the single most important parameter of the Standard Model. The Qweak experiment will be a low-energy measurement of the weak mixing angle, which is a test of the Standard Model running of the angle [3, 4]. As shown in Fig. 1 the value of the weak mixing angle is predicted to change (in the $\overline{\mathrm{MS}}$ renormalization scheme) by $\sim 4 \%$ from the energy scale of the $Z$ pole (where a decade of precision measurements has been made at high energy colliders at SLAC and CERN [5]) to the low energy scale of Qweak. With a $0.3 \%$ measurement of the weak mixing angle, the Qweak experiment will make a $\sim 10 \sigma$ verification of this effect.

Weak charge and mixing angle results can also be obtained in other types of experiments. In atomic parity violation experiments $[6,7,8]$, weak charge values are complicated to extract due to atomic structure and many-body nuclear effects (see, for example $[9,10])$. The $\mathrm{NuTeV}$ experiment [11] at Fermilab has seen evidence of 
a non-Standard Model value for the weak mixing angle at a lower energy scale than the $Z$ pole, but its result is sensitive to choice of parton distributions and radiative corrections. A currently running experiment at SLAC, E158 [12], is measuring parity violation in electron-electron scattering, and is complementary to Qweak in its sensitivity to different types of new physics (for example: SUSY, leptoquarks, $Z^{\prime}$ boson, R-parity violation, ... ) [3, 4]. The scientific impact of the Qweak experiment will be: a direct measurement of the proton's weak charge in a simple system; a theoretically clean method of measuring precisely the weak mixing angle at low energy scale, a relatively unexplored regime of the Standard Model; and a possible unambiguous indication of new physics.

\section{THE QWEAK EXPERIMENT}

An illustration of the conceptual design of the Qweak experiment is shown in Fig. 2. The experiment consists of scattering of a longitudinally polarized $1.2 \mathrm{GeV}$ electron beam by a $35 \mathrm{~cm}$ liquid hydrogen target. Elastically scattered electrons at $9 \pm 2$ degrees are selected by a collimation system, and the electrons are focused by a large toroidal magnet onto a set of eight synthetic quartz Cerenkov detectors. At the average experimental momentum transfer of $Q^{2}=0.03 \mathrm{GeV}^{2}$ the expected Qweak asymmetry is small, -0.3 parts per million (ppm). The expected event rate for scattered electrons of $\sim 6 \mathrm{GHz}(760 \mathrm{MHz}$ per detector octant) precludes counting the individual events. Instead, the experiment will use current mode detection and low noise front end electronics.

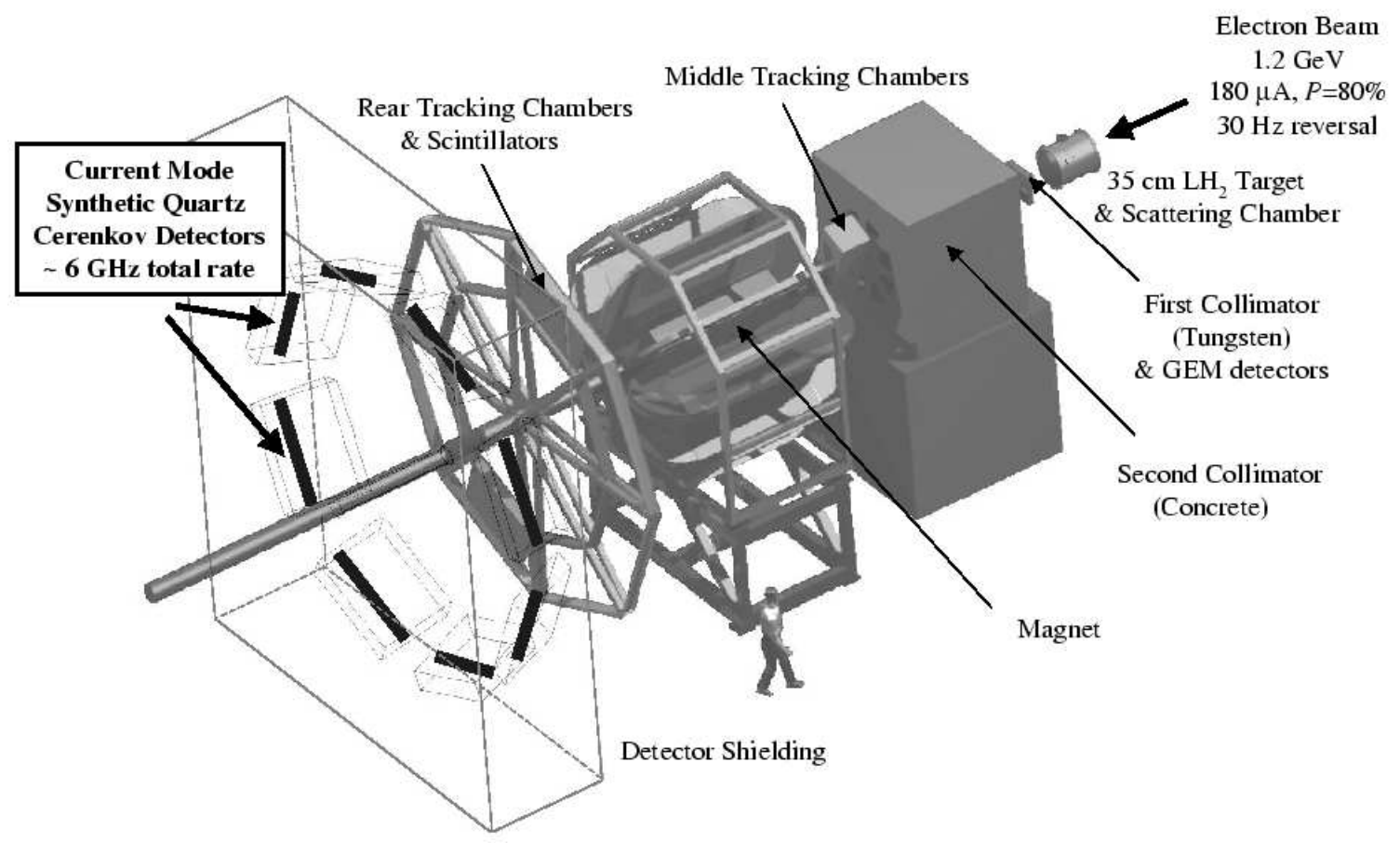

Figure 2: Conceptual design for the Qweak experimental setup, in Hall C at Jefferson Lab. The eight quartz detectors are each $2 \mathrm{~m} \times 12 \mathrm{~cm} \times 2.5 \mathrm{~cm}$. The spectrometer provides clean separation of elastic and inelastic electrons at its focal plane. 
A tracking system will be used with the beam current reduced by four orders of magnitude, allowing individual events to be observed. This will enable both a measurement of the dilution of the Cerenkov detector signal by background and a precise determination of the average $Q^{2}$. The tracking system components will rotate to cover all octants, and will include the following three sets of detectors: a gas electron multiplier as a front vertex detector; wire chambers near the magnet entrance as a measure of scattering angle; and, at the focal plane, vertical drift chambers to allow mapping of the analog response of the Cerenkov system, and large scintillators as a charged particle trigger.

To extract the physics of interest for Qweak requires an extrapolation to low $Q^{2}$ of hadronic form factors, $B\left(Q^{2}\right)$ above, which will be the dominant systematic error for the experiment at $2 \%$. These form factors will contribute approximately one third of the experimental asymmetry. They are known with reasonable precision at higher $Q^{2}$, and are suppressed by a factor of $Q^{2}$ with respect to the weak charge contribution for Qweak. Other experiments completed or underway at JLab $\left(\mathrm{G}^{0}\right.$, HAPPEX, HAPPEX II), Bates at MIT (SAMPLE), and MAMI at Mainz (A4), will be used to constrain these form factors. If necessary, in the future the Qweak collaboration could pursue an independent measure of $B\left(Q^{2}\right)$ by running at a $Q^{2}$ different from $0.03 \mathrm{GeV}^{2}$.

Precision beam polarimetry is required in order to have a polarization contribution to systematic error of less than $1.5 \%$, and a Compton polarimeter will be constructed in JLab Hall C. Radiative corrections for the polarized $e-p$ scattering process at these kinematics have been calculated and are known with a precision of $0.8 \%$ [3].

The Qweak physics proposal was approved by the January 2002 JLab PAC with an 'A' scientific rating, and Qweak has become an important new thrust of the JLab scientific program. The experiment presented a successful technical design review in January 2003. The Qweak experiment will proceed in two stages: a statistics limited run with a low power target to achieve an $8 \%$ or better result on the asymmetry in 2007, followed by a run of 2200 hours at $180 \mu \mathrm{A}$ to achieve a $4 \%$ result. The absolute limits of the technique are under study. This research was supported in part by the U.S. Department of Energy, the National Science Foundation, and the Natural Sciences and Engineering Research Council of Canada.

\section{References}

[1] Qweak Collaboration: Carlini, R. et al., Jefferson Lab Proposal E-02-020. Proposal available at http://www.jlab.org/qweak/.

[2] Musolf, M.J. et al., Phys. Rep. 239, 1 (1994).

[3] Erler, J., Kurylov, A., and Ramsey-Musolf, M.J., Phys. Rev. D68, 016006 (2003).

[4] Kurylov, A., Ramsey-Musolf, M.J., and Su, S., hep-ph/0303026, to appear in Phys. Rev. D. 
[5] Particle Data Group: Hagiwara, K. et al., Phys. Rev. D66, 010001 (2002). In particular, the section Electroweak Model and Constraints on New Physics.

[6] Edwards, N.H. et al., Phys. Rev. Lett. 74, 2654 (1995).

[7] Vetter, P.A. et al., Phys. Rev. Lett. 74, 2658 (1995).

[8] Wood, C.S. et al., Science 275, 1759 (1997).

[9] Kuchiev, M.Y. and Flambaum, V.V., Phys. Rev. Lett. 89, 283002 (2002).

[10] Milstein, A.I., Sushkov, O.P., and Terekhov, I.S., Phys. Rev. Lett. 89, 283003 (2002).

[11] NuTeV Collaboration: Zeller, G.P. et al., Phys. Rev. Lett. 88, 091802 (2002).

[12] SLAC-E-158 Collaboration: Hughes, E.W., Kumar, K.S., Souder, P.A., et al., SLAC-Proposal-E-158.

$\dagger$ The Qweak Collaboration:

D.S. Armstrong ${ }^{1}$, T.D. Averett ${ }^{1}$, J. Birchall ${ }^{2}$, J.D. Bowman ${ }^{3}$, R.D. Carlini ${ }^{4}$, S. Chattopadhyay ${ }^{4}$, C.A. Davis ${ }^{5}$, J. Doornbos ${ }^{5}$, J.A. Dunne ${ }^{6}$, R. Ent ${ }^{4}$, J. Erler ${ }^{7}$, W.R. Falk ${ }^{2}$, J.M. Finn ${ }^{1}$, T.A. Forest ${ }^{8}$, D.J. Gaskell ${ }^{4}$, K.H. Grimm ${ }^{1}$, C. Hagner ${ }^{9}$, F.W. Hersman ${ }^{10}$, M. Holtrop ${ }^{10}$, K. Johnston ${ }^{8}$, R.T. Jones ${ }^{11}$, K. Joo ${ }^{11}$, C. Keppel ${ }^{12}$, E. Korkmaz ${ }^{13}$, S. Kowalski ${ }^{14}$, L. Lee ${ }^{2}$, A. Lung ${ }^{4}$, D. Mack ${ }^{4}$, S. Majewski ${ }^{4}$, G.S. Mitchell ${ }^{3}$, H. Mkrtchyan ${ }^{15}$, N. Morgan ${ }^{9}$, A.K. Opper ${ }^{16}$, S.A. Page ${ }^{2}$, S.I. Penttila ${ }^{3}$, M. Pitt ${ }^{9}$, M. Poelker ${ }^{4}$, T. Porcelli ${ }^{13}$, W.D. Ramsay ${ }^{2}$, M.J. Ramsey-Musolf ${ }^{17}$, J. Roche ${ }^{4}$, N. Simicevic ${ }^{8}$, G.R. Smith ${ }^{4}$, R. Suleiman ${ }^{14}$, S. Taylor ${ }^{14}$, W.T.H. van Oers ${ }^{2}$, S.B. Wells ${ }^{8}$, W.S. Wilburn ${ }^{3}$, S.A. Wood ${ }^{4}$, C. Zorn ${ }^{4}$

${ }^{1}$ College of William and Mary, Williamsburg, Virginia 23187

${ }^{2}$ University of Manitoba, Winnipeg, MB, Canada R3T 2N2

${ }^{3}$ Los Alamos National Laboratory, Los Alamos, New Mexico 87545

${ }^{4}$ Thomas Jefferson National Accelerator Laboratory, Newport News, Virginia 23606

${ }^{5}$ TRIUMF, Vancouver, BC, Canada V6T 2A3

${ }^{6}$ Mississippi State University, Mississippi 39762

${ }^{7}$ Instituto de Física, Universidad Nacional Autónoma de México, 04510 México D.F., México

${ }^{8}$ Louisiana Technical University, Ruston, Louisiana 71272

${ }^{9}$ Virginia Polytechnic Institute and State University, Blacksburg, Virginia 24061

${ }^{10}$ University of New Hampshire, Durham, New Hampshire 03824

${ }^{11}$ University of Connecticut, Storrs, Connecticut 06269

${ }^{12}$ Hampton University, Hampton, Virginia 23668

${ }^{13}$ University of Northern British Columbia, Prince George, BC, Canada V2N 4 Z9

${ }^{14}$ Massachusetts Institute of Technology, Cambridge, Massachusetts 02139

${ }^{15}$ Yerevan Physics Institute, 375036 Yerevan, Armenia

${ }^{16}$ Ohio University, Athens, Ohio 45701

${ }^{17}$ California Institute of Technology, Pasadena, California 91125 